\title{
CIENCIAS DE LA SALUD
}

\section{Percepción sobre la calidad de vida en mujeres con mastectomías del Hospital San Pablo de Asunción}

\author{
Carmelo Maidana Pereira¹, Ingrid Melgarejo Ruíz Díaz
}

\begin{abstract}
Resumen
Introducción: Esta investigación está enfocada en la percepción que se tiene sobre la calidad de vida de las mujeres con mastectomías. La calidad de vida es la percepción que tiene el paciente en las circunstancias en que se encuentre, los valores que desarrolle, la satisfacción a sus necesidades, y bienestar; los riesgos a los que se somete su salud física y mental, siendo importante el cómo afrontan esta situación, que dependerá de la resolución de sus problemas, sus expectativas, su estado emocional, de su entorno para poder compensar cualquier tipo de daño percibido.
\end{abstract}

Objetivo: Determinar la calidad de vida en mujeres con mastectomía, del Hospital San Pablo de Asunción.

Material y Método: La investigación que se realizó es de tipo descriptivo, bibliográfico, documental, estadístico, retrospectivo porque se utilizaron datos estadísticos e historias clínicas de pacientes con mastectomías. Se indagó sobre el tema en varios libros, diccionarios y páginas web autorizadas. Se utilizó la Medición. Las técnicas e instrumentos fueron la observación indirecta de casos en fichas clínicas. Este estudio se realizó a 100 mujeres mastectomizadas sobre la percepción de su condición física y la necesidad de una calidad de vida agradable y llevadera.

Resultados: El tipo detectado con mayor frecuencia es el total con $75 \%$, el lado predominante afectándose más la mama izquierda con 44\%, el lado derecho con $39 \%$ y ambas mamas el $17 \%$. Con relación a las características clínicas las pacientes reportaron presencia de hipertensión arterial en el 52\%, malignidad previa $28 \%$ y diabetes mellitus $20 \%$. El $72 \%$ de las pacientes se encontraba en la fase curativa y el $28 \%$ en la etapa paliativa con metástasis y recaída de la enfermedad. El $65 \%$ de las pacientes recibió como tratamiento complementario quimioterapia y el $35 \%$ radioterapia. El $36 \%$ de las pacientes cree gozar de salud muy buena, el $30 \%$ cree que es buena, el $17 \%$ de las pacientes dijeron

1. Universidad Iberoamericana. Facultad de Ciencias de la Salud, Paraguay.

E-mail: carmelomaidanapereira@gmail.com

DOI: $10.26885 /$ rcei.foro.2019.119

Trabajo publicado en acceso abierto bajo Licencia Creative Commons. 
que tienen una salud excelente, el $12 \%$ cree que su salud es regular, y el $5 \%$ cree que es mala. Las enfermedades crónicas degenerativas como el cáncer, enmarcan un problema de salud pública mundial debido al incremento anual de morbimortalidad. En las mujeres con mastectomía, la calidad de vida se ve afectada según los años que se encuentre el paciente tras la cirugía y el tratamiento que reciba, nos muestra una serie de respuestas psicosociales que limita su afrontamiento adecuado ante la situación. El principal hallazgo en esta investigación fue la alta valoración en las escalas de la salud general y calidad de vida de los pacientes con cáncer de mama, que dieron como resultado un $36 \%$ que valora su salud general como muy buena y el $30 \%$ valora como buena su calidad de vida.

Conclusiones: A nivel general, las pacientes con mastectomías reportan que la enfermedad y el tratamiento médico no han interferido demasiado en la realización de las actividades de la vida diaria, en actividades o encuentros de tipo social o familiar, como tampoco interfirió en la capacidad para concentrarse o recordar los acontecimientos de su entorno.

Palabras clave: mastectomías, cáncer, percepción.

\section{Referencias}

Arraras, J. I., Martínez, M., Manterota, A., Laínez, N. (2004). La evaluación de la calidad de vida del paciente oncológico: el grupo de calidad de vida de la EORTC.

Bloom, J., Fotopoulis, S., Flame,r G., Holland, J. C., Muenz, L., Murawski, B. (2019). Psychological response to mastectomy.A prospective Comparison study.

Casso, D., Buist, D. S., Taplin, S. Quality of life of 5-10 year breast cancer survivors diagnosed between age 40 and 49.

OMS. (sf). Cáncer de mama: prevención y control. Washington: OMS. http:// www.who.int/topics/cancer/breastcancer/es/.

. Plumb, M, M., Holland, J. (1977). Comparative studies of psychological function in patient with advanced cancer- I. Self- reported depressive symptoms. Psychosom. Med., 39(4), 264-7. 